\title{
Applications of particle tracking velocimetry to severe nuclear accident experimentation
}

\author{
M. Johnson ${ }^{1}$, C. Journeau ${ }^{1} *$ \\ ${ }^{1}$ CEA, DES, IRESNE, DTN, SMTA, LEAG, Cadarache, 13115 Saint-Paul-lez-Durance, France \\ * christophe.journeau@cea.fr
}

\begin{abstract}
Experimental research into severe nuclear accidents may entail the discharge of a very high-temperature lava-like molten fuel mixture, corium, either into a pool of less-dense, more-volatile coolant or onto a solid substrate where the corium will spread and cool. In both instances, remote, high-speed video imaging is usually required to interpret these transient interactions and PTV represents a powerful tool for the characterisation of the dynamic properties of discrete melt fragments or distinctive features in the surface of the melt during spreading. Nuclear fuel-coolant interactions present particular challenges for PTV analysis as a molten jet and its fragments can exhibit high rates of inter-frame deformation and undergo fragmentation with a relatively high frequency. A PTV algorithm, adapted to these challenges, is presented whereby a user-defined tolerance in the evolution of certain particle properties is used to refine the potential candidate particles prior to particle matching. This candidate refinement step is used to distinguish between acceptable levels of deformation between successive sightings of a given particle, and more substantial changes consistent with fragmentation or coalescence, requiring the tracking of a new particle. Implementation of the PTV algorithm is presented for (1) an X-ray video from the FCINA-30-1 experiment between a jet of molten stainless steel and liquid sodium, conducted at the JAEA's MELT facility, and (2) video imaging of the VE-U9-ceramic experiment of a molten corium-thermite mixture spreading on a zirconium substrate, conducted at the CEA's VULCANO facility. The latter case-study enabled the characterization of $>70,000$ local velocity vectors at locations corresponding to distinctive temperature heterogeneities in the surface of the spreading melt, providing extensive insight into the spreading dynamics for the validation of corium spreading models.
\end{abstract}

\section{Introduction}

During a hypothetical core disruptive accident (CDA) in a nuclear reactor, nuclear fuel and core infrastructure would rapidly melt leading to the relocation of molten core material, a high-temperature lava-like substance referred to as corium. One possible progression of the CDA concerns the discharge of coherent jets of molten nuclear fuel through core discharge tubes, designed to reduce reactivity in the core (Bertrand et al. (2018)), or control rod guide tubes, to a pool of the less dense, more volatile coolant, leading to a fuelcoolant interaction (FCI). This phase of the CDA is of interest to nuclear engineers as the fine fragmentation of the molten fuel, sudden destabilisation of vapour films surrounding melt fragments and rapid heat transfer between melt and coolant raises the potential for vapour explosions (Berthoud (2000)).

A subsequent phenomenon of interest concerns the possibility that the reactor pressure vessel is breached by the high-temperature corium, resulting in corium discharge to the containment floor (Dinh et al. (2000); Journeau et al. (2003)). Under these circumstances it is desirable to promote ex-vessel spreading over a broad surface area to enhance the dissipation of radioactive decay heat and preserve the integrity of the containment building (Johnson et al. (2021a)). Both of these hypothetical scenarios have been the subject of extensive experimental research. The JAEA's MELT facility (Matsuba et al. (2016); Emura et al. (2019); Johnson et al. (2021b)) and CEA's KROTOS facility (Zabiego et al. (2010); Tyrpekl et al. (2014)) have been used to investigate FCIs for sodium-cooled and water-cooled reactor configurations respectively, using X-ray imaging to visualise the interactions within the optically opaque test vessels (Berge et al. (2018); Johnson et al. (2021b)). The CEA's VULCANO facility has been employed to investigate the discharge of molten corium-thermite mixtures and their subsequent spreading on concrete or ceramic substrates (Journeau et al. 
(2003, 2006); Johnson et al. (2021a)). Mutliple optical and infra-red cameras are used to observe the progression of the melt during spreading.

Particle tracking velocimetry (PTV) (Crocker and Grier (1996); Brevis et al. (2011); Ohmi and Li (2000)) is a powerful tool for the interpretation of images acquired during $\mathrm{FCI}$ and corium spreading experiments. Particle-matching enables analysis of the population of melt fragments without the double counting bias of the multiple sightings of each particle. The estimated velocity of missile fragments and the rate of expansion of vapour clouds could also be used to infer the energetics of fragmentation events. The spreading velocity of an advancing melt front and local velocity distribution similarly provide crucial insight into melt spreading dynamics for the validation of severe accident codes (Spindler and Veteau (2006); Wittmaack (2002)).

Each type of experiment presents unique challenges for PTV analysis. The hydrodynamic pre-mixing of a molten metallic jet in a less dense fluid represents a complex three-dimensional transient interaction, captured by a single two-dimensional projection of the attenuated fan-beam X-ray source. The interaction occurs within a timescale of a few seconds, during which the particle population evolves significantly. A fragmentation event generates multiple potential candidate particles in close proximity, the new trajectories of which may either be tracked as a continuation of the original particle or characterized as new particles depending on the rigidity particle-matching criteria. Coalescence events present similar complications, particularly as these can be difficult to distinguish from quasi-coalescence whereby distinct particles temporarily by-pass each other at different depths in the X-ray photon path. Different FCI experimental facilities generate X-ray images with frame rates ranging from 100-1000 fps (Zabiego et al. (2010); Emura et al. (2019); Johnson et al. (2021b)) and the size of imaging fields of view vary significantly, requiring that the PTV algorithm tolerates a wide range of particle densities and offers flexibility in the particle matching criteria to account for the different rates of inter-frame particle deformation. A PTV algorithm is presented whereby candidate particle pairings are assessed in terms of a weighted similarity index enabling the rejection of candidate particles of insufficient likeness to the paired sighting prior to particle matching. The application of this PTV approach is demonstrated for (1) the FCINA-30-1 FCI experiment (Johnson et al. (2021b)) between a jet of molten steel and liquid sodium, performed at the MELT facility, and (2) the VE-U9-ceramic experiment (Johnson et al. (2021a)) of a corium-thermite melt spreading on a zirconium substrate, performed at the VULCANO facility.

\section{Experimental methods}

\subsection{Fuel-coolant interaction experiments}

FCI experimental facilities, such as KROTOS and MELT, consist, in simplest terms, of a crucible for the melting of metal and metal oxide nuclear fuel analogues, a funnel to discharge the melt as a coherent jet, a test section containing a pool of liquid coolant, and a radiographic imaging system to visualise the interaction within the optically opaque environment. To demonstrate the application of PTV to FCI experimental images, the FCINA-30-1 experiment (Johnson et al. (2021b)) conducted at the JAEA's MELT facility (Matsuba et al. (2016); Emura et al. (2019)) will be investigated. This experiment was conducted between a jet of molten stainless steel and a pool of sodium coolant. A detailed explanation of the MELT facility and experimental procedure can be found in Johnson et al. (2021b). The radiographic images were acquired at a frame rate of $1000 \mathrm{fps}$ for a field of view in the order of $\sim 12 \mathrm{~cm}$ in diameter (Johnson et al. (2021b)). This FCI research at the MELT facility was performed under the framework of the current implementing arrangement of the France-Japan collaboration on Sodium-cooled Fast Reactors (SFRs) from 2020 to 2024.

\subsection{Ex-vessel corium spreading experiments}

The VULCANO facility consists of a zirconium crucible with a discharge tube at its base, suspended above a spreading test section with boundary walls which diverge at an angle of $9.5^{\circ}$. The VE-U9 spreading tests discharge $\mathrm{a} \approx 30 \mathrm{~kg}$ molten corium-thermite mixture to spreading test sections with either a ceramic zirconium or sacrificial concrete substrate. The spreading is imaged from multiple perspectives using optical and infrared cameras stored in protective housings. Complete details of the experimental procedure are presented in Johnson et al. (2021a). 


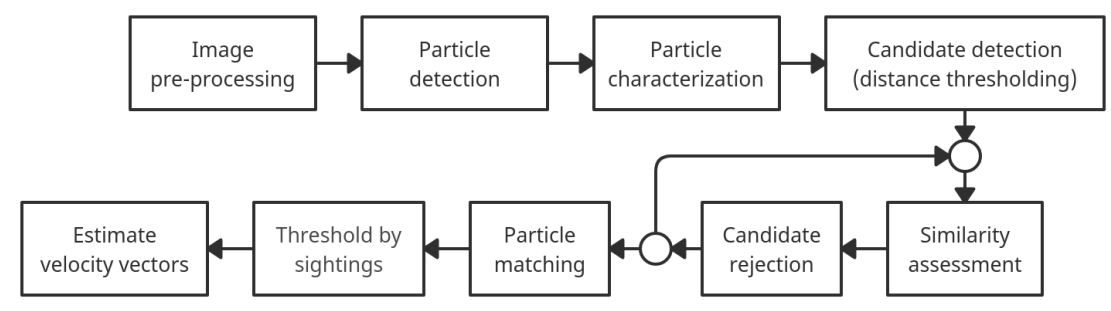

Figure 1: Flow chart for the PTV algorithm.

\subsection{Image processing and analysis}

The images generated during FCI and ex-vessel spreading experiments are processed and subsequently analysed using SPECTRA (Software for Phase Extraction and Corium TRacking Analysis), a CEA image analysis software developed using Matlab 2021a (Mathworks, USA) (Johnson et al. (2021b)). SPECTRA is used to (1) clean the images of artefacts, such as additive white noise, lens distortion and vignetting, (2) extract objects of interest for analysis, and (3) perform quantitative characterization of the detected objects, including the implementation of tracking by PTV.

\subsubsection{Particle tracking velocimetry}

The PTV framework implemented in SPECTRA, presented as a flow chart in Figure 1, is based on the algorithm proposed in Crocker and Grier (1996) for images of colloidal particles but uses an alternative approach for particle matching more specific to the requirements of FCI experimental images. The objective of the alternative particle matching routine is to allow the user to define bespoke, video-specific criteria to distinguish minor particle deformations from more significant fragmentation or coalescence events which constitute the formation of a new particle.

Following image pre-processing and segmentation, all detected particle sightings are identified, labelled and characterized according to $K$ digital particle properties, not limited to the centroid, area, perimeter, caliper dimensions, orientation, mean and maximum intensity, aspect ratio and circularity. The PTV algorithm proceeds with the pre-selection of candidate particles, which is performed by distance thresholding. The centroid coordinates of $j$ candidate particles in frame $i+1\left(x_{i+1, j}\right)$ will have an Euclidean distance from a particle centroid in frame $i$ of less than a defined maximum displacement, $R,($ Ohmi and Li (2000)) therefore satisfying the inequality:

$$
\left|x_{i+1, j}-x_{i}\right| \leq R
$$

Candidate particle assessment is performed using the $K$ properties of each digital particle. The corresponding $k^{\text {th }}$ property of the current frame particle and the $j$ candidate particles are labelled $y_{i, k}$ and $y_{i+1, j, k}$ respectively. Each property is assigned a weighting coefficient, $\omega_{k}$, to refine the array of particle properties to a single coefficient called a weighted similarity index, WSI, calculated according to eq. 2 .

$$
W S I=\frac{1}{\sum_{k=1}^{K} \omega_{k}} \sum_{k=1}^{K} \omega_{k} \frac{\left|y_{i, k}-y_{i+1, j, k}\right|}{y_{i, k}} \leq M W S I
$$

The WSI diminishes with increased likeness between the current frame and candidate particle. If only a single property, such as the area, is assigned a non-zero weighting coefficient, the WSI represents the percentage change in that property between consecutive video frames, normalised by a factor of 100 . The candidate particles are refined by eliminating those with a WSI greater than a threshold maximum weighted similarity index, MWSI. The MWSI defines an acceptable tolerance in the evolution in particle properties between consecutive frames. This MWSI coefficient should be set to allow for sufficient tolerance in both the real particle deformation between frames and the more artificial uncertainties in particle detection and characterization due to imaging artefacts. The candidate particle assessment and refinement steps can be performed iteratively using different $\omega_{k}$ and $M W S I$ values to eliminate candidate particles which fail to satisfy alternative similarity criteria.

The remaining candidate particles, which have satisfied the requisite conditions of proximity and physical likeness, proceed to the particle matching stage. The current frame particle and candidate particle pair 


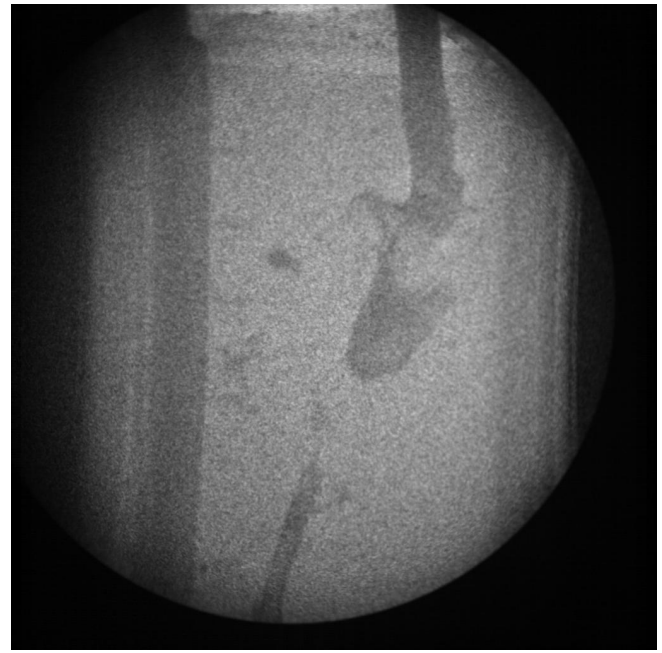

(a)

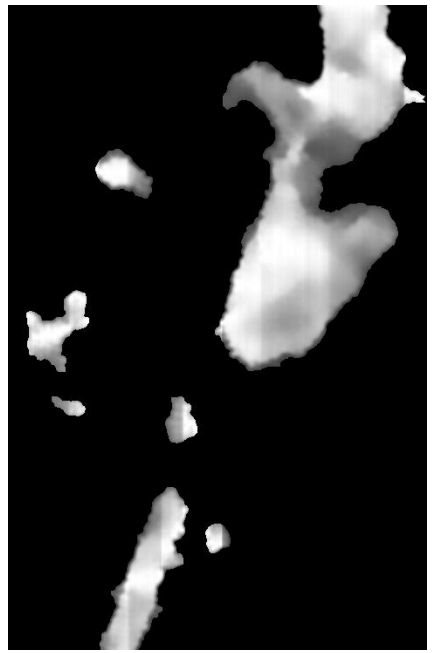

(b)

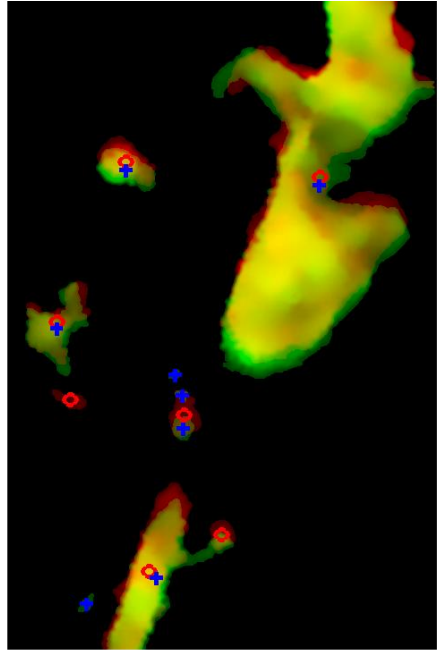

(c)

Figure 2: (a) An example X-ray image from the FCINA-30-1 experiment (reproduced from Johnson et al. (2021b)), (b) the same image following pre-processing and melt segmentation, cropped to a region of interest, and (c) a semi-transparent overlay of (b) and the subsequent frame with the centroids indicated as red circles (current frame) and blue crosses (subsequent frame).

with the lowest WSI are assigned the same particle number and are subsequently removed from alternative candidate particle pairings. The process is repeated until no valid particle pairs remain within the two images under consideration. The algorithm repeats this process for the subsequent image pairs. On completion of the particle matching and labelling process, unlabelled particles and particles which present with fewer than the desired minimum sightings, are removed. Particles are then renumbered sequentially, if preceding particle numbers have been eliminated, and the characterization data is sorted by ascending particle number and then by frame number.

The final stage requires the estimation of velocity vectors. In most cases the velocity vectors are determined from the Euclidean displacement of the centroid, or intensity weighted centroid, normalised by the frame interval. For objects cropped at the image border, such as a molten jet entering the imaging window from above, the centroid displacement does not provide an accurate measure of the trajectory. For these border objects, the compass points of a rectangular bounding box around the object are defined and the displacement of the coordinates opposite the cropped boundary (i.e. the southern coordinate of the bounding box for objects entering from the top of the image) are used to estimate the particle trajectory.

\section{Results and discussion}

\subsection{Fuel-coolant interactions}

An example X-ray image from the FCINA-30-1 experiment, reproduced from Johnson et al. (2021b), is presented in Figure $2 \mathrm{a}$. The image suffers from some common X-ray imaging artefacts, such as loss of intensity towards the image border (vignetting) and considerable additive white noise. The images are denoised in two stages using a spatiotemporal video-block matching and 3D filter, V-BM3D, (Danielyan et al. (2012)), followed by a non-local means, NLM, (Buades et al. (2005)) filter to remove residual noise. The images are then normalised by a clean flat field image, acquired from the averaged frames prior to the arrival of the melt jet, and corrected for pincushion distortion. The pre-processed images are then dynamically thresholded and cropped to a region of interest below the initial surface of the sodium pool and within an internal cylinder within the test section. Application of this image pre-processing and segmentation routine to the image in Figure $2 \mathrm{a}$ generates the image in Figure $2 \mathrm{~b}$. A compound image of the melt fragments in Figure $2 \mathrm{~b}$ with a semi-transparent overlay of those observed in the subsequent frame, with their centroids shown as red circles and blue plus signs respectively, is presented in Figure $2 \mathrm{k}$.

These image data have a number of advantageous qualities for exploitation by PTV. The images are 


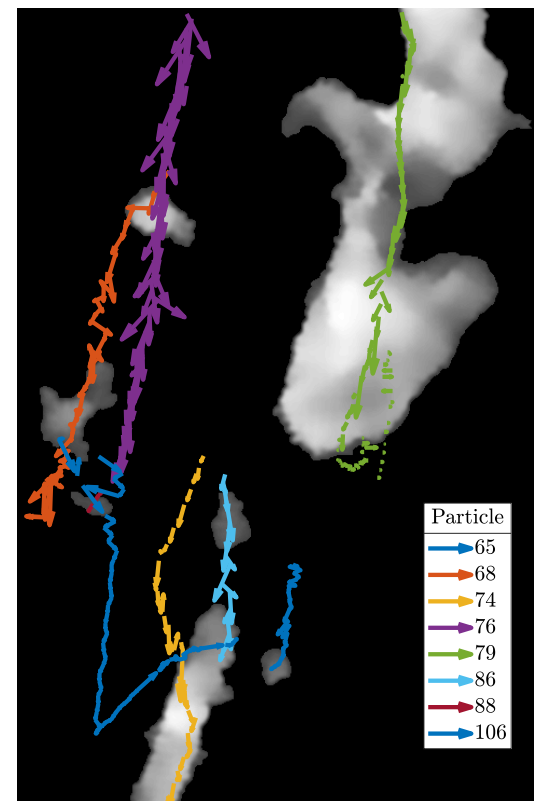

(a)

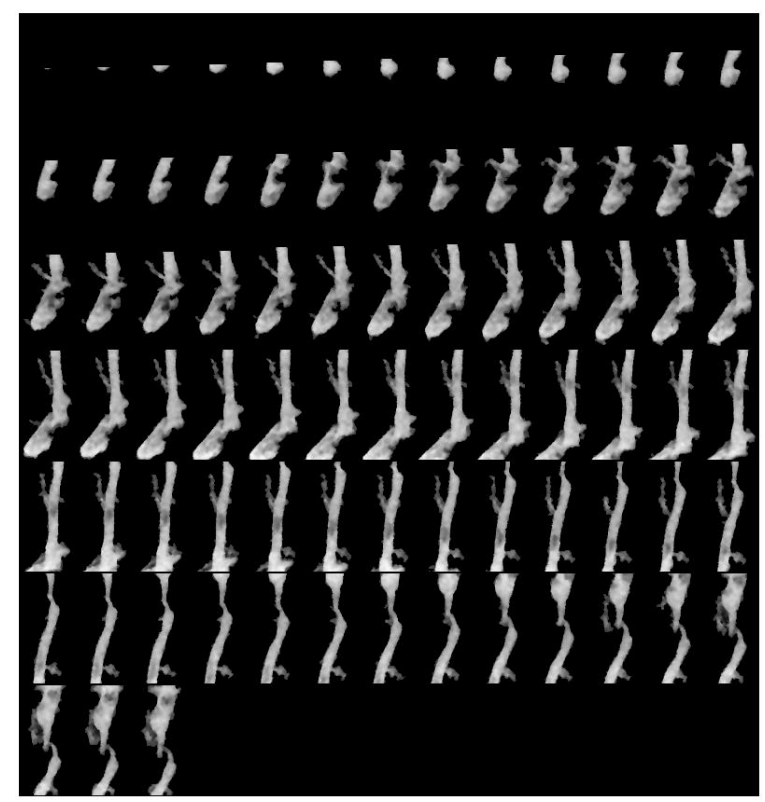

(b)

Figure 3: (a) The segmented image from Figure $2 \mathrm{p}$ overlaid with velocity vectors revealing the complete trajectories of each particle, and (b) a chronological montage of all sightings of the coherent jet (particle 79) prior to its fragmentation.

high in resolution giving a relatively low particle density of $\sim 10^{-5}$ pixel $^{-1}$. The particles are macroscopic, morphologically distinctive, and are often relatively simple to distinguish. The high frame rate dictates a relatively low inter-frame particle displacement, often significantly smaller than the particle dimensions, and so particle pairings are typically self-evident. The complexity in applying PTV arises from (1) high rates of particle deformation due their molten nature, (2) a high frequency of particle division and coalescence, (3) apparent or quasi-coalescence due to the flattening of the depth field in the 2D image projection, (4) the partial or total obscuration of some particles by vapour clouds, (5) the possible detection of false particles due to the significant additive white noise in the raw images, and (6) the fact that the interaction extends beyond the limits of the field of view, thereby cropping many sightings at the image border. Figure $2 \mathrm{c}$ identifies seven distinct first frame melt sightings, with eight sightings observed in the subsequent frame. Of the seven first frame particle sightings, two are cropped at the image border, entering and leaving the imaging window respectively, four sightings appear to coalesce to form two particles in the subsequent frame, one particle fragments into two daughter particles of similar dimensions, and two sightings in the second frame appear to be false particles detected due to the image noise.

For these X-ray projections of melt fragments, the intensity profile of the particle is proportional to the particle dimension, or chord length, parallel to the photon flux (Macovski (1983)), thus, small particles may present with a very low signal to noise ratio. Despite the implementation of powerful image denoising filters, the detection of sub-millimetre particles will generally necessitate the detection of some false particles, corresponding to small pockets of residual noise. Since these false particles present at random spatiotemporal locations, rather than in close proximity to previous sightings, an initial iteration of the PTV algorithm with a conservative (relatively large) maximum displacement and a minimum sightings threshold of at least 2 will eliminate the majority of false particles. These false particles can be effaced from the video in a PTV-segmentation step, prior to a second, more precise iteration of the PTV algorithm with more stringent tracking parameters. Coupling the conventional intensity and size based thresholding of image segmentation with the distance and similarity thresholding of this PTV-segmentation step to eliminate false particles can thereby enable particle detection at a reduced signal to noise ratio. Implementation of the PTV algorithm with a maximum displacement of 50 pixel and a minimum sightings threshold of 2 , with no similarity threshold $(M W S I \rightarrow \infty)$, eliminates 1398 false particles from the population of 10422 .

Refining the tracking parameters to a maximum displacement of 35 pixel, allowing for a $20 \%$ tolerance in the change in particle area between successive frames (this tolerance is relaxed for objects entering and 
leaving the imaging window) and a minimum sightings threshold of 5 identifies 338 particles with 4547 sightings. Thus, roughly half the total number of objects detected in the video correspond to long-life particles presenting with, on average, 13 sightings per particle. All of the velocity vectors corresponding to the 7 particles observed in Figure $2 \mathrm{~b}$, and one additional particle (particle 106) produced following a quasi-coalescence involving particle 76, are presented, overlaid on the segmented image, in Figure 3 a. The tracking history of particles 68 and 76 reveals a shared origin which implies particle 68 to be a daughter particle following the fragmentation of particle 76. The most interesting observation from Figure 3 a concerns the sudden reversal in the trajectory of particle 106 close to the bottom left corner of the image. Closer inspection of the video revealed that the particle in question experiences an elastic collision with a thermocouple installed on the steel support rod observed in the left hand side of Figure $2 \mathrm{a}$. The elastic collision not only infers solid-like behaviour, confirming the formation of a solid outer crust at the melt-coolant interface, but indicates that within $90 \mathrm{~ms}$ of contact with the coolant, the melt crust grows to a sufficient thickness to withstand $\mathrm{a} \approx 0.9 \mathrm{~m} \mathrm{~s}^{-1}$ collision with the thermocouple without fracturing.

Figure $3 \mathrm{~b}$ presents a chronological montage of all sightings of particle 79 , the coherent melt jet, prior to its fracture. The morphology of the jet similarly exhibits solid-like qualities within $\approx 20 \mathrm{~ms}$ of contact with the sodium. After around $40 \mathrm{~ms}$ of entering the imaging window, the jet spans the height of the imaging window. While the PTV algorithm continues to recognise the image spanning jet as the same particle, the characterization of the velocity vectors ceases to function for the image-spanning sightings since the centroid, intensity weighted centroid and the coordinates of the bounding box all become misleadingly static and fail to accurately capture the inter-frame displacement. For objects which span the length of the imaging window an alternative method will be required for the spatial cross-correlation of adjacent sightings to determine the particle trajectory. A normalized 2D cross-correlation (Lewis (1995)) approach is currently being implemented in SPECTRA for the determination of the trajectories of these selected image spanning particles.

\subsection{Ex-vessel spreading}

Figure 4 a presents an example image from the VE-U9-ceramic melt spreading experiment (Johnson et al. (2021a)). The melt is segmented from each image by dynamic thresholding and a mask of the spreading section floor is used to refine the melt to its footprint. Corners of the squares in the checkerboard image in Figure $4 \mathrm{p}$ are defined as control points, used to generate a transform function to correct the camera perspective to a bird's-eye view of the melt. The product of these segmentation and transformation steps is presented in Figure $4 \mathrm{c}$.

Application of PTV to the melt footprint in Figure $4 \mathrm{c}$ enables characterization of the velocity of the advancing melt front as presented in Johnson et al. (2021a), however this provides only limited insight into the spreading dynamics. The melt surface appears highly heterogeneous in temperature, providing a distinctive pattern in the melt luminosity. Convolution of the image with an $11 \times 11$ pixel Laplacian of Gaussian (LoG) operator with a standard deviation of 2, highlights the features with a significant change in local luminosity, corresponding mainly to the outer melt contour and a series of local heterogeneities in the melt surface temperature, generally features of reduced luminosity implying cold-spots, as presented in Figure $4 \mathrm{~d}$. Size thresholding of the features to retain those comprised of 5-50 pixels isolates the localised cold-spots from the larger features. PTV is employed to track these surface features and reveal the evolution of the local surface velocity distribution with time and space.

Application of the LoG edge detection and size thresholding to the VE-U9-ceramic experimental images revealed a total of 96755 surface features. Modeling of the VE-U9 spreading dynamics in Johnson et al. (2021c) predicted surface velocities of up to around $0.25 \mathrm{~m} \mathrm{~s}^{-1}$. The maximum velocity, combined with a pixel resolution in the transformed image of around $1.6 \mathrm{~mm}$ and a frame rate of $50 \mathrm{fps}$, dictates that local surface features are unlikely to move more than 4 pixel between consecutive frames. This implies that many features will exhibit displacements of less than a pixel and so velocity vectors will need to be averaged over multiple frames for accurate representation.

The PTV algorithm was employed with a maximum displacement of 6 pixel, allowing for a $20 \%$ change in the area and mean intensity of features between consecutive frames, and a minimum sightings threshold of 5. A total of 71527 sightings were found for 4534 distinct features, enabling the characterization of local velocity vectors at $74 \%$ of the locations where surface temperature heterogeneities were detected. The instantaneous local velocities are averaged over five frames (or $0.1 \mathrm{~s}$ intervals) to smooth the data and to detect sub-pixel displacements. The local velocities are presented for the frames at 2 and $5.7 \mathrm{~s}$ after the initial melt discharge to the spreading section in Figures 4 e and $\mathrm{f}$ respectively. While a maximum velocity, parallel to the principal flow direction, $v_{x}$, was found to be $0.45 \mathrm{~m} \mathrm{~s}^{-1}, 98 \%$ of the velocity vectors in the principal 


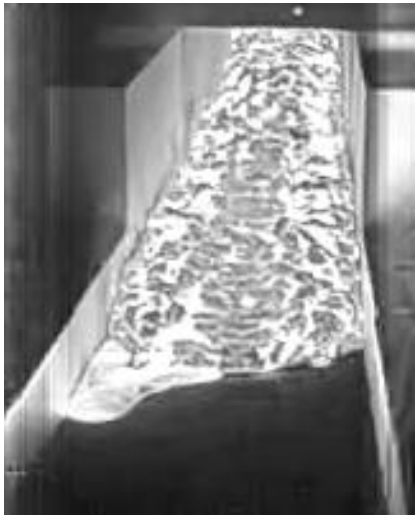

(a)

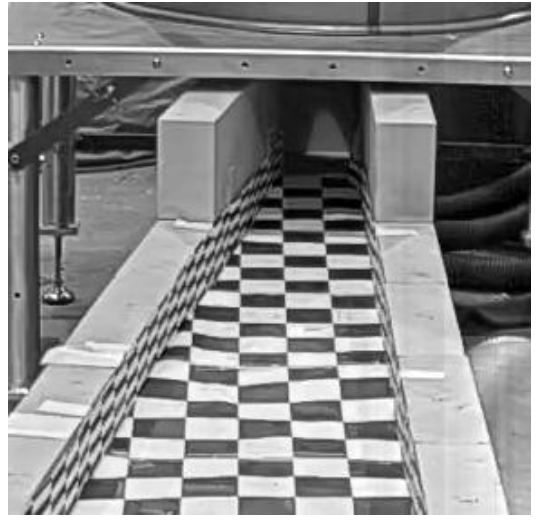

(b)

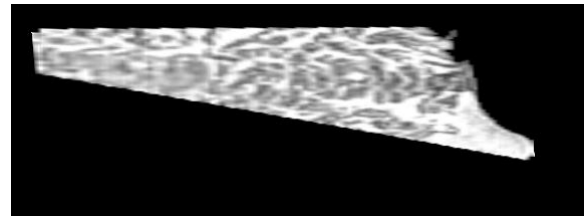

(c)

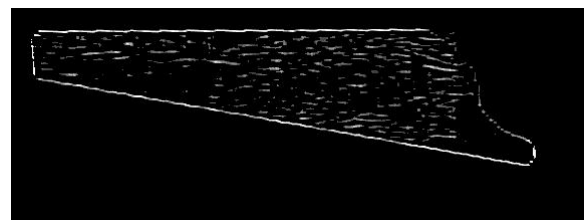

(d)

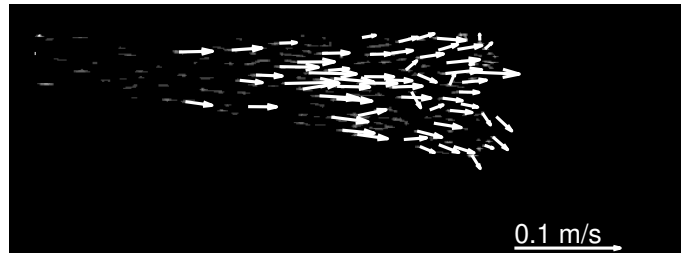

(e)

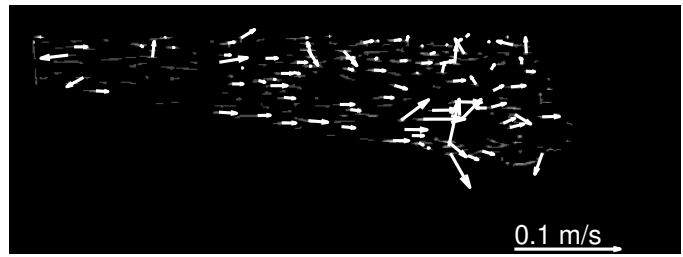

(f)

Figure 4: Images of (a) corium-thermite spreading on a zirconium substrate during the VE-U9-ceramic test, (b) a checkerboard calibration image of $5 \times 5 \mathrm{~cm}$ squares, (c) the segmented melt from (a), transformed to a birds-eye perspective, (d) a Laplacian of Gaussian transform of (c) revealing the melt contour and temperature heterogeneities in the melt surface, and (e-f) quiver plots of the PTV-determined velocity vectors after (e) $2 \mathrm{~s}$ and (f) $5.7 \mathrm{~s}$; the images in (c-f) correspond to dimensions of $830 \times 340 \mathrm{~mm}$.

flow direction were less than $0.23 \mathrm{~m} \mathrm{~s}^{-1}$, which demonstrates good agreement with theoretical predictions from Johnson et al. (2021c).

\section{Conclusions}

Experimentation in the field of severe nuclear accident research often requires remote video imaging of hightemperature transient interactions. PTV represents a very powerful tool for the analysis of (1) radiographic images acquired during investigations of molten fuel-coolant interactions, and (2) optical and infrared images acquired of ex-vessel melt spreading. Fuel-coolant interactions present particular challenges for PTV analysis as the molten fuel exhibits high rates of deformation and a high frequency of fragmentation. A bespoke PTV algorithm is presented whereby candidate particles are refined by user-defined similarity criteria prior to particle matching, enabling the distinction between the tolerable deformation of a molten fuel droplet and a more significant fragmentation event which constitutes the formation of new particles.

Application of the PTV algorithm to the FCINA-30-1 fuel-coolant interaction experiment (Johnson et al. (2021b)) between molten stainless steel and liquid sodium enabled the detection of 338 relatively long-life particles presenting with an average of 13 sightings each. One melt particle presented with a sudden reversal in its trajectory which revealed an elastic collision with a thermocouple installed within the test section. This distinctly solid-like behavior confirms that within $90 \mathrm{~ms}$ of first contact with the liquid sodium, the steel formed a solid crust at the melt-coolant interface of sufficient thickness to withstand a $0.9 \mathrm{~m} \mathrm{~s}^{-1}$ collision with the thermocouple, implying a crude lower limit for the rate of crust growth. Certain of the tracked particles, representing large melt jet fragments with strong evidence of a frozen outer shell, spanned the height of the imaging window and inhibited the characterisation of velocity through the displacement of the centroid or the leading front. The PTV algorithm will require an alternative cross-correlation technique to estimate the motion of objects with dimensions greater than the field of view. A second application of the PTV algorithm to images acquired during the VE-U9-ceramic spreading test (Johnson et al.(2021a)) enabled 
the tracking of distinctive surface temperature heterogeneities, or cold-spots, in the molten corium-thermite mixture during its spreading on a ceramic substrate. This enabled the characterisation of over 70,000 local velocities during the $\approx 10$ s spreading test, providing extensive data for the validation of ex-vessel corium spreading simulations.

\section{Acknowledgements}

The authors would like to thank the JAEA, in particular everybody from the MELT team, for sharing their FCINA-30-1 experimental data under the France-Japan collaboration on the SFR severe accident studies. We also wish to thank Mitsubishi Heavy Industries for their financing of the VE-U9 spreading tests in collaboration with the CEA, and the CEA's PLINIUS team for their management of the VULCANO facility during the VE-U9 tests.

\section{References}

Berge L, Estre N, Tisseur D, Payan E, Eck D, Bouyer V, Cassiaut-Louis N, Journeau C, Tellier RL, Singh S, and Pluyette E (2018) Fast high-energy X-ray imaging for severe accidents experiments on the future PLINIUS-2 platform. IEEE Transactions on Nuclear Science 65:2573-2581

Berthoud G (2000) Vapor explosions. Annual Review of Fluid Mechanics 32:573-611

Bertrand F, Marie N, Bachrata A, Brun-Magaud V, Droin J, Manchon X, Herbreteau K, Farges B, Carluec B, Poumerouly S, and Lemasson D (2018) Status of severe accident studies at the end of the conceptual design of ASTRID: Feedback on mitigation features. Nuclear Engineering and Design 326:55-64

Brevis W, Niño Y, and Jirka GH (2011) Integrating cross-correlation and relaxation algorithms for particle tracking velocimetry. Experiments in Fluids 50:135-147

Buades A, Coll B, and Morel JM (2005) A review of image denoising algorithms, with a new one. Multiscale Modeling Simulation 4:490-530

Crocker JC and Grier DG (1996) Methods of digital video microscopy for colloidal studies. Journal of Colloid and Interface Science 179:298-310

Danielyan A, Katkovnik V, and Egiazarian K (2012) BM3D frames and variational image deblurring. IEEE Transactions on Image Processing 21:1715-1728

Dinh TN, Konovalikhin MJ, and Sehgal BR (2000) Core melt spreading on a reactor containment floor. Progress in Nuclear Energy 36:405-468

Emura Y, Isozaki M, Matsuba K, and Kamiyama K (2019) Penetration behavior of molten stainless steel into a sodium pool. in Proceedings of the 24th national symposium on power and energy systems (SPES 2019). page 583

Johnson M, Denoix A, Bouyer V, Goda H, Kamohara SS, Takeuchi J, Brissonneau L, and Journeau C (2021a) High-temperature ex-vessel corium spreading. part 1: Experimental investigations on ceramic and sacrificial concrete substrates. submitted to Journal of Nuclear Science and Technology

Johnson M, Journeau C, Matsuba K, Emura Y, and Kamiyama K (2021b) Characterization of hightemperature nuclear fuel-coolant interactions through X-ray visualization and image processing. Annals of Nuclear Energy 151:107881

Johnson M, Schiano T, Denoix A, Bouyer V, and Journeau C (2021c) High-temperature ex-vessel corium spreading. part 2: Scaling principles for gravity-viscous spreading with slip at the melt-substrate interface. submitted to Journal of Nuclear Science and Technology

Journeau C, Boccaccio E, Brayer C, Cognet G, françois Haquet J, Jégou C, Piluso P, and Monerris J (2003) Ex-vessel corium spreading : results from the vulcano spreading tests. Nuclear Engineering and Design 223:75-102 
Journeau C, Haquet JF, Spindler B, Spengler C, and Foit J (2006) The VULCANO VE-U7 corium spreading benchmark. Progress in Nuclear Energy 48:215-234

Lewis JP (1995) Fast template matching. in Vision Interface. pages 120-123

Macovski A (1983) Medical imaging systems. Prentice-Hall. 1st edition

Matsuba KI, Isozaki M, Kamiyama K, and Tobita Y (2016) Distance for fragmentation of a simulated molten-core material discharged into a sodium pool. Journal of Nuclear Science and Technology 53:707712

Ohmi K and Li HY (2000) Particle-tracking velocimetry with new algorithms. Measurement Science and Technology 11:603-616

Spindler B and Veteau JM (2006) The simulation of melt spreading with thema code part 1 : Model assessment strategy and assessment against analytical and numerical solutions. Nuclear Engineering and Design 236:415-424

Tyrpekl V, Piluso P, Bakardjieva S, and Dugne O (2014) Material effect in the nuclear fuel-coolant interaction: Analyses of prototypic melt fragmentation and solidification in the KROTOS facility. Nuclear Technology 186:229-240

Wittmaack R (2002) Simulation of free-surface flows with heat transfer and phase transitions and application to corium spreading in the EPR. Nuclear Technology 137:194-212

Zabiego M, Brayer C, Grishchenko D, baptiste Dajon J, Fouquart P, Bullado Y, Compagnon F, Correggio P, françois Haquet J, and Piluso P (2010) The KROTOS KFC and SERENA/KS1 tests: experimental results and MC3D calculations. pages 1-11 